\title{
Segmentation of Left Ventricle via Level Set Method Based on Enriched Speed Term
}

\author{
Yingge Qu, Qiang Chen, Pheng Ann Heng, and Tien-Tsin Wong \\ Department of Computer Science and Engineering \\ The Chinese University of Hong Kong, Shatin, Hong Kong \\ ygqu@cse. cuhk. edu.hk
}

\begin{abstract}
Level set methods have been widely employed in medical image segmentation, and the construction of speed function is vital to segmentation results. In this paper, two ideas for enriching the speed function in level set methods are introduced, based on the problem of segmenting left ventricle from tagged MR image. Firstly, a relaxation factor is introduced, aimed at relaxing the boundary condition when the boundary is unclear or blurred. Secondly, in order to combine visual contents of an image, which reflects human visual response directly, a simple and general model is introduced to endow speed function with more variability and better performance. Promising experimental results in MR images are shown to demonstrate the potentials of our approach.
\end{abstract}

\section{Introduction}

Computer-aided diagnosis is an important application domain of medical image analysis. Segmentation and tracking of cardiac structures are advanced techniques used to assist physicians in various states of treatment of cardiovascular diseases.

There have been many methods for segmentation purpose. Snake-driven approaches [1] are popular in medical image segmentation; however it cannot perform well around a cusp or in topology changing cases. B-splines, deformable templates, and Fourier descriptors are common ways to describe the structure of interest. Level set representation is an emerging technique to represent shapes and track moving interfaces [5]. Such techniques are applicable to segmentation and tracking 4. Dealing with local deformations, multicomponent structures and changes of topology are the main strengths of these representations.

In the level set method, the construction of speed function is vital to the final result. The speed function is designed to control the movement of curve; and in different application problems, the key is to determine the appropriate stopping criteria for the evolution [6]. In case of segmentation, the segmentation precision depends on when and where the evolving curved surface stops, and the termination of the evolving curved surface also depends on the speed term. So the construction of speed term is critical to the segmentation results.

In this paper, two ideas for enriching the speed function are introduced, based on the problem of segmenting left ventricle from tagged MR image. Relaxation 
factor is first introduced. It provides a force to stop evolving curve at ventricle boundary even when the boundary is blurred. As visual contents of image, including color, shape and texture reflect human visual response directly, various image content based speed terms have been introduced to tackle different application problems. Here, we derive a simple and general model, through introducing the image content items, to endow speed function with more variability and better performance.

The rest of this paper is organized as follows. In Section 2, level set method and segmentation procedure are introduced. Section 3 focuses on the speed term improvement and presents experimental results. Conclusion is given in Section 4

\section{Level Set Method and Left Ventricle Segmentation}

\subsection{Level Set}

Level set method, developed by Osher and Sethian, is a zero equivalent surface method [5]. Its basic idea is to change movement track of planar curve into movement track of three-dimensional curved surface. Though this conversion complicates the solution, it has many other merits; and the main merit is that it can deal with the change of topological structure easily.

The classical level set boundary is defined as the zero level set of an implicit function $\Phi$ defined on the entire image domain. The dimensionality of the level set function $\Phi$ is one higher than the evolving boundary, which in this case for a 2-D image domain is a 3-D surface. The level set method tracks the evolution of a front that is moving normal to the boundary with a speed $F(x, y)$. The speed function can be dependent on the local or global properties of the evolving boundary or from forces external to the boundary. The level set function $\Phi$ is defined such that the location of the boundary $\Gamma$ and the region enclosed by the boundary $\Omega$ are functions of the zero-level set of $\Phi$, namely,

$$
\Phi_{t}+F|\nabla \Phi|=0 \text {. }
$$

It is the basic equation of level set, and the zero level set denotes object contour curve

$$
\Gamma(t)=\{x \mid \Phi(x, t)=0\} .
$$

Generally, $\Phi$ represents the signed distance function to the front. That is,

$$
\Phi(x, y)= \pm d(x, y)
$$

where $d(x, y)$ is the smallest distance from the point to the boundary, and the sign is chosen such that points inside the boundary have a negative sign and those outside have a positive sign.

The function $\Phi$ is initialized based on a signed distance measure to the initial front. In the case of a single point this is simply the euclidian distance to that point. The evolution of the boundary is defined via a partial differential equation on the zero level set of $\Phi$

$$
\frac{\partial \Phi}{\partial t}=-F|\nabla \Phi|
$$


Finally, level set methods may be easily adapted to a discrete grid implementation for efficient processing. Refer to [6] for a more detailed description on level set methods and their applications.

\subsection{Left Ventricle Segmentation}

The level set framework has been adapted to a number of applications beyond front propagation of physical phenomena, including the denoising of images, object recognition, and shortest path identification. Key to the success of these approaches is to determine the appropriate stopping criteria for the evolution [6]. The level set will continue to propagate as long as the speed function $F(x, y)>0$. Therefore, the speed function must be properly designed such that $F(x, y) \rightarrow 0$ as the boundary approaches the desired location. For the segmentation problem, it is desirable for $F(x, y) \rightarrow 0$ at the edges of the left ventricle boundary. The algorithm flow is:

1. Initialize the narrow band;

2. Resolve the level set equation (4);

3. Track the zero level set curve, and reinitialize the narrow band;

4. Repeat step 3 and 4 , and enter the calculations of the next time step.

The Hamilton-Jacobi equation (4) can be resolved according to the hyperbolic conservation law. When the curve evolves to the boundary of the narrow band, the narrow band should be reinitialized. If the point difference between sequential times is smaller than the designed threshold in narrow band, the propagating speed will be set to zero. When the narrow band is not reinitialized within some time, it means that the curve evolvement has stopped. So the iteration ends, and the final zero level curve is the boundary of the left ventricle.

\section{Improvement on Speed Term}

Much of the challenge in interface problems comes from producing an adequate model for speed term [6]. As mentioned above, the construction of speed function is vital to final results. The speed function is designed to control the movement of curve; and in different problems, the key is to determine the appropriate stopping criteria for the evolution. In a segmentation case, the segmentation precision depends on when and where the evolving curved surface stops, and the stop of the evolving curved surface also depends on the speed term $F$. So the construction of $F$ is critical to the segmentation results.

In this section, based on the problem of segmenting left ventricle from tagged MR image, two ideas to enrich the speed function are introduced. Relaxation factor is first introduced, followed by an introduction to image content items.

\subsection{Relaxation Factor}

In 4], the construction of speed term is described as: $F=F_{A}+F_{G}$, where $F_{A}$ is a constant. It does not depend on the geometry of the front, but its sign 
determines the movement direction of the front. $F_{G}$ depends on the geometry of the front. It is ignored in this case, so supposing $F_{G}=0$. In [4] a negative speed term is constructed as equation (5), and then constructs the speed term as: $F=F_{A}+F_{I}$, where $F_{I}$ is defined as

$$
F_{I}(x, y)=\frac{-F_{A}}{M_{1}-M_{2}}\left\{\left|\nabla G_{\sigma} \cdot I(x, y)\right|-M_{2}\right\} .
$$

The expression $G_{\sigma} \cdot I(x, y)$ denotes the image convolved with a Gaussian smoothing filter whose characteristic width is $\sigma . M_{1}$ and $M_{2}$ are the maximum and minimum values of the magnitude of image gradient $\left|\nabla G_{\sigma} \cdot I(x, y)\right|$. So the speed term $F$ tends to be zero when image gradient is large.

However, in practical, gradient on object boundary are impossible of the same maximum value $\left(M_{1}\right)$. In other word, the evolvement cannot stop at the object boundary. Especially for MR images whose boundaries are blurry, the results are more unsatisfactory. To solve this problem, here, a relaxation factor $\delta$ is introduced to relax the bounding of $M_{1}-M_{2}$ :

$$
r=\frac{\left|\nabla G_{\sigma} \cdot I(x, y)\right|-M_{2}}{M_{1}-M_{2}-\delta}
$$

where $\delta \in\left[0, M_{1}-M_{2}\right]$. Let $r=\left\{\begin{array}{l}r \text { if } r<1 \\ 1 \text { if } r \geq 1\end{array}\right.$, and the reconstructed negative speed term will be $F_{I}^{\prime}(x, y)=-r \cdot F_{A}$.

By introducing this relaxation factor, when the front propagates to places where the gradient of object boundary are close to $M_{1}$, but not exactly $M_{1}$, the speed $F$ drops to zero and the evolving curve will be stopped soundly. Fig. 1 shows the result of solving this problem by introducing the relaxation factor $\delta$. Here, $\delta=0.23$.

\subsection{Image Content Items}

Visual content of an image includes color, shape, texture, and spatial layout, reflecting human visual response directly. Based on them, image/object feature extraction techniques have been fully developed. In typical level set methods, the construction of speed function mainly uses the gradient information. However, as medical imaging technique advances, more clear and detailed images have brought more difficulties to level set based segmentation methods. As a response, various image content based speed items have been introduced to solve different application problems. In this paper, we derive a simple yet general model, through combining the image content items, to endow the speed function with more variability and better performance.

The form of speed function can be written as [4]: $F=F_{A}+F_{G}$. By adding control items or other image force, it can be expressed as a general but simple model:

$$
\begin{aligned}
& F=A\left(F_{A}+F_{G}\right)+B \\
& A=C_{\text {img }} / C_{\text {front }} \text { or } A=1-\operatorname{dist}\left(C_{\text {img }}, C_{\text {front }}\right) \\
& B=\operatorname{dist}\left(C_{\text {img }}, C_{\text {front }}\right)
\end{aligned}
$$




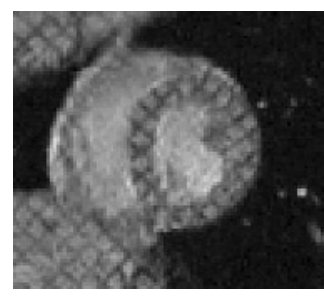

(a)

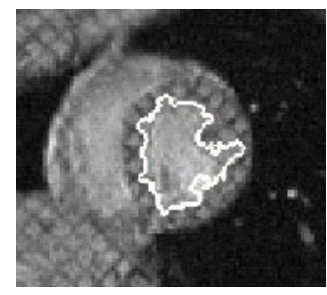

(b)

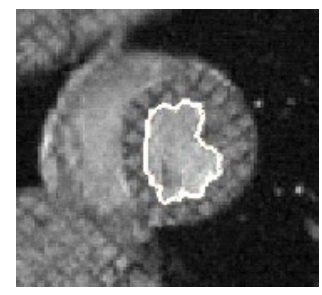

(c)

Fig. 1. (a) is the original MR image. In (b), the white line is the propagated front get by original level set speed term. It easily gets across the true boundary due to the blurred boundary. (c) is the result by introducing the relaxation factor into the speed term.

The $\operatorname{dist}()$ could be an arbitrary distance function. It is engaged to measure the difference of image features between the propagating front and the target image. $B$ stands for the additional forces come from image content. $C$ stands for the image content model, including any of color, shape, and texture features. $A$ is used to balance the force from front and the force from additional items, and $A \rightarrow 1$.

\subsection{The Enriched Speed Terms}

For example, when curve movement is relative to curvature, namely $F_{G} \neq 0$, literatures [4] and 8] introduce the stop term based on image gradient:

$$
K_{I}(x, y)=\frac{1}{1+\left|\nabla G_{\sigma} \cdot I(x, y)\right|}
$$

Here, gradient can be treated as the difference measure of gray/brightness value. To adjust the image gradient's influence on the speed term, we can overwrite it:

$$
K_{I}^{\prime}(x, y)=\frac{1}{1+\left|\nabla G_{\sigma} \cdot I(x, y)\right|^{p}}=1-\frac{\left|\nabla G_{\sigma} \cdot I(x, y)\right|^{p}}{1+\left|\nabla G_{\sigma} \cdot I(x, y)\right|^{p}},
$$

where the constant $p \geq 1$. When $p$ is larger, $K_{I}^{\prime}$ will be smaller, so it will control the speed to decrease faster. Then the speed term can be written as

$$
F=K_{I}^{\prime}\left(K+F_{A}\right)
$$

where $K=\frac{\phi_{y y} \phi_{x}^{2}-2 \phi_{x} \phi_{y} \phi_{x y}+\phi_{x x} \phi_{y}^{2}}{\left(\phi_{x}^{2}+\phi_{y}^{2}\right)^{3 / 2}}$ is the curvature of the front at point $(x, y)$.

Other examples: To resolve boundary leak problem, Kichenassamy et al. [2] and Yezzi et al. 9] introduced a pull-back term $(\nabla c \cdot \nabla \phi)$ due to edge strength, here $c(x, y)=\frac{1}{1+\left|\nabla G_{\sigma} \cdot I(x, y)\right|}$. It is an additional force term based on the color feature. To provide an additional attraction force when the front was in the vicinity of an edge, Siddiqui et al. 7] introduced an extra term $\frac{1}{2} d i v\left[\left(\begin{array}{l}x \\ y\end{array}\right) \phi\right]$ 
according to the area minimization. It is an additional force term based on the shape feature.

In tagged MR image case, new feature also can be introduced based on this idea. High clarity of tag lines makes segmentation more complex than untagged image. According to human response, texture is the most distinguish feature, see Fig. 2(a). In this example, the tag lines are on vertical direction, hence we design an additional term $B$ based on texture feature $B P V[3]$.

$$
B P V(x, y)=\frac{1}{N} \sum_{y-\left\lceil\frac{N-1}{2}\right\rceil}^{y+\left\lceil\frac{N-1}{2}\right\rceil}(I(x, i)-M)^{2}, M=\frac{1}{N} \sum_{y-\left\lceil\frac{N-1}{2}\right\rceil}^{y+\left\lceil\frac{N-1}{2}\right\rceil}|I(x, i)-I(x, y)|
$$

Here, the distance is measured by the standard deviation. $B P V(x, y)$ is the $B P V$ value of point $(x, y), 1 \leq x \leq m, \frac{N-1}{2} \leq y \leq n-\frac{N-1}{2}, m$ and $n$ are height and width of image, and $N$ is the width of window, which is appropriate to be 7 or 9 according to the width of tag lines. $I(x, y)$ is the intensity value of the block center. Meaning of the above equation is: When the intensity value of some point is quite different from that of points which are on the same line and whose center is the point, this $B P V$ value of the point is smaller, and vice verse. Now, the speed function has been enriched to:

$$
F=K_{I}^{\prime}\left(\varepsilon K+F_{A}+F_{I}^{\prime}\right)+\beta \frac{\nabla c \cdot \nabla \phi}{|\nabla \phi|}+\mu \operatorname{div}\left[\left(\begin{array}{l}
x \\
y
\end{array}\right) \phi\right]+\gamma(B P V),
$$

where $K_{I}^{\prime}$ is the $A$ item, used to control the balance between the original speed term and the additional speed term. Larger $K_{I}^{\prime}$ will speedup the decrease of the speed term. The coefficients $\beta, \mu$, and $\gamma$ are constant, which should be defined according to the contribution of their following part. In equation (14), image features, such as curvature, shape and texture information, have been combined into the speed term. Experiment with this enriched speed item is carried in the following section.

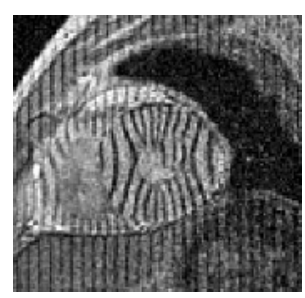

(a)

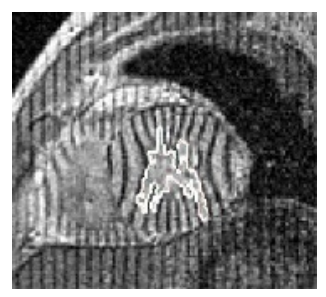

(b)

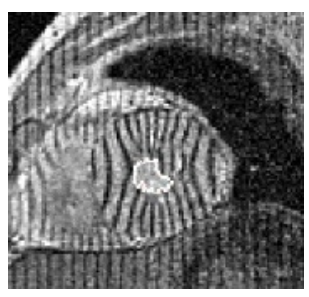

(c)

Fig. 2. (a) shows the strong tagged MR image (b) is the result obtained by the standard level set method. This result has a serious leak phenomenon due to the affection from strong tag lines. (c) is the improved result by using the BPV feature to control the propagation of the front. 


\subsection{Experiments and Results}

The first experiment focused on the left ventricle MR image with tag lines. Because of the blood flow, tag lines within the left ventricle decrease fast in the last stage of the cardiac shrink, until they disappear; But tag lines in the myocardium decrease slowly, which can be seen from Fig. 2(a). In other words, within the left ventricle the intensity changes are smaller and the relative BPV value is larger; in the myocardium the intensity changes are larger and the relative BPV value is smaller. As for images like Fig. 2(a) whose tag lines are very strong, the weight of BPV should be larger in the construction of speed term.

In the above experiment, the spatial step length is 1 , the temporal step length is $0.001, F_{A}=-20, \beta=5, \mu=0.0025, \gamma=280, \delta=0.23$. The number of iterations is 150. As the tag lines in Fig. 2 are strong, constant $\gamma$ should be set large. While in other cases, it could be set small. For MR images without tag lines, we do not have to impose the BPV term (namely $\gamma=0$ ). These parameters are fine tuned empirically and adjusted manually. An automatic process to estimate the parameters could be further studied in future work [10].

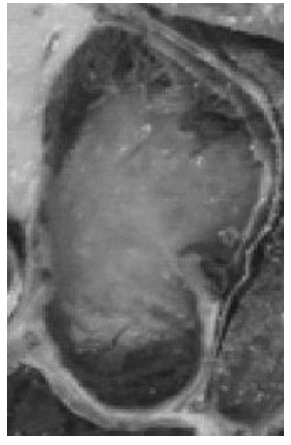

(a)

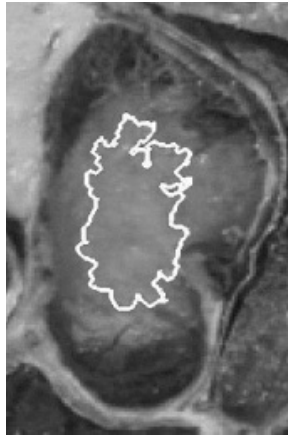

(b)

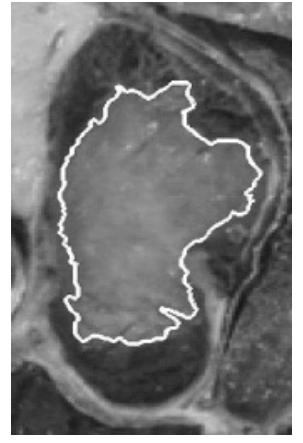

(c)

Fig. 3. (a) is the original color slide image. (b) shows that propagate front stop at the non-boundary area. (c) is the result after incorporating color and texture features into the speed function.

This model can be applied to various cases. For example, in color slide image case, another experiment result can be seen in Fig. 3. The original segmentation result is far from ideal (Fig. 3(b)) because that the stronger image details could stop the propagation easily. In this case, the introduction of color and texture into the speed term makes the result closer to user's expectation. So we introduce block color histogram and $B P V$ texture feature. The distance measures are: the histogram intersection, as a measure of the block wised histogram; and the Euclidean distance, as a measure in $B P V$ item. Balancing items are: $F_{A}=-15$, $\beta=5, \mu=0.002, \gamma=20, \delta=0.2$. 


\section{Conclusion}

In medical image segmentation problems, image features vary much. Gradient and gray level information are far from enough to describe proper stopping condition of the speed term. Introducing new speed item to control the propagating is necessary and important. In this paper, two ideas for enriching the speed function are introduced. Relaxation factor is first introduced. It provides a relaxing boundary condition, so as to stop the evolving curve at a blurred or unclear boundary. Secondly, the speed term is enriched by introducing visual content items. A simple and general model is proposed to incorporate the image features into the speed item in order to improve the flexibility and performance of the speed function. Promising experiment shows that the incorporation of powerful distance measure and proper image features in speed function can improve the segmentation result significantly.

Acknowledgement. This work was supported by the Research Grants Council of the Hong Kong Special Administrative Region, under RGC Earmarked Grants (Project CUHK 4180/01E, CUHK 1/00C).

\section{References}

1. M. Kass, A. Witkin, and D. Terzopoulos. Snakes: Active contour models. IJCV, pages 321-332, 1988.

2. S. Kichenassamy, A. Kumar, P. Olver, A. Tannenbaum, and A. Yezzi. Conformal curvature flows: from phase transitions to active vision. Archive of Rational Mechanics and Analysis, 134(3):275-301, 1996.

3. C. G. Looney. Pattern Recognition Using Neural Networks, chapter 10. Oxford University Press, 1997.

4. R. Malladi, J. Sethian, and B. Vemuri. Shape modeling with front propagation: A level set approach. IEEE Pattern Anal. Machine Intell., 17:158-175, 1995.

5. Stanley Osher and James A. Sethian. Fronts propagating with curvature-dependent speed: algorithms based on hamilton-jacobi formulations. J. Comput. Phys., 79(1):12-49, 1988.

6. J.A. Sethian. Level Set Methods and Fast Marching Methods: Evolving Interfaces in Computational Geometry, Fluid Mechanics, Computer Vision, and Materials Science. Cambridge University Press, 1999.

7. K. Siddiqui, Y.B. Lauriere, A. Tannenbaum, and S. W. Zucker. Area and length minimizing flows for shape segmentation. IEEE Trans. on Image Processing, $7(3): 433-443,1998$.

8. J. Suri, K. Liu, S. Singh, S. Laxminarayana, and L. Reden. Shape recovery algorithms using level sets in 2-d/3-d medical imagery: A state-of-the-art review. IEEE Trans. in Information Technology in Biomedicine (ITB), 6(1):9-12, 2001.

9. A. Yezzi, S. Kichenassamy, A. Kumar, P. Olver, and A. Tannenbaum. A geometric snake model for segmentation of medical imagery. IEEE Trans. on Medical Imaging, 16(2):199-209, 1997.

10. A. J. Yezzi, A. Tsai, and A. S. Willsky. Binary and ternary flows for image segmentation. In Proc. Int. Conf. on Image Processing, volume 2, pages 1-5, 1999. 